\title{
Habitus outsider
}

\author{
Vanessa RIPIO RODRÍGUEZ \\ Universidad Complutense de Madrid \\ vaneripio@gmail.com
}

Recibido: Mayo 2015

Aceptado: Junio 2015

\section{RESUMEN}

El grupo social se apropia de las y los agentes mediante un proceso de mimesis corporal. El sentido práctico queda así incorporado. Pierre Bourdieu lo denomina habitus y, del mismo, forman parte los esquemas prácticos de la dominación masculina. Los experimentos creadores de las outsiders, propuestos por Virginia Woolf en Tres Guineas, podrían romper con esa sociodicea masculina no quebrantando las leyes sino hallando la ley.

Palabras clave: Habitus, Campo, Violencia simbólica, Experimento, Arte.

\section{Outsider habitus}

\begin{abstract}
The social group appropriates the agents via a process of corporal mimesis. The practical sense is in this way incorporated. Pierre Bourdieu called it habitus and the practical schemes of male dominance form part of it. The Outsiders creative experiments, suggested by Virginia Woolf in Three Guineas, destined not to break the law, but find the law, should break down with the male-dominated society.
\end{abstract}

Keywords: Habitus, Field, Symbolic Violence, Experiment, Art.

\section{INTRODUCCIÓN}

La expresión que da título al presente artículo, outsider habitus, es un completo contrasentido. El primer término es el que Virginia Woolf utiliza en Three Guineas para describir el particular estatus al que las mujeres hijas de caballeros han de aspirar en la sociedad inglesa de los tardíos años 30. El segundo, es una de las nociones centrales de la investigación sociológica llevada a cabo por Pierre Bourdieu durante la segunda mitad del siglo pasado. Por eso, lo primero será iniciarnos en la teoría bourdiana y en la peculiar etnología wolffiana para entender no solo el contrasentido en cuestión, sino el sentido que queremos dar aquí al ineludible problema. 
Habitualmente, la filosofía ha considerado el cuerpo como un elemento separado y sospechoso, peligroso para el ejercicio de la teoría, asunto por excelencia de las almas. Este dualismo, históricamente arraigado y aún en absoluto superado, está sin duda vinculado a la dicotomía radical con la que los esquemas prácticos de la dominación masculina dividen el mundo, segregando la componente femenina de la masculina, considerando el primero como el polo negativo, dominado y pasivo de la cosmogonía social que todavía pervive en nuestro mundo social.

El cuerpo cayó muy pronto de ese lado oscuro, húmedo y opaco, dominado por indómito, perseguido por místicos y teóricos, para ser luego recuperado como la sede de los placeres del paraíso perdido y, en particular, cómo enseña Foucault, de una sexualidad igual de opaca que urge desencadenar como lo propiamente humano y dónde libertad y felicidad pueden por fin reunirse la una con la otra. Pero esta apoteosis, cuento de hadas revolucionario de las nuevas masculinidades del siglo pasado, no ha dejado de conservar la dicotomía original, ni la función reproductora del eterno masculino, ni la necesidad de reiterar la superioridad del depredador, del dominante, en definitiva de un juego de suma cero que solo tiene sentido si el uno es más que la otra. $\mathrm{Y}$ esa renovada unidad ganadora, sádica y masculinizada sigue teniendo al cuerpo como otredad perdedora, victimizada y feminizada, como artículo fundamental del mercado de la violencia del juego del patriarcado. Comprender cómo funciona y para qué sirve el cuerpo cuando intermedia esa violencia, no solo ejercida con/contra los cuerpos sino también con/contra los cerebros, conforma la primera parte de este artículo.

Pensamos aquí que aunque esa emergencia política de los cuerpos pudiera verse como un paso necesario para pensar más allá de los esquemas de la dominación masculina, seguir hablando únicamente del cuerpo conserva en lo esencial la dicotomía que los fundamenta. Si hay cuerpo a secas es porque en algún lugar, quizá no consciente, oculto, sigue habiendo alma, espíritu, mente y con ello, la relación jerárquica de control y dominación que los define. Reaccionar a ella y reivindicar el elemento dominado cómo el auténtico, continúa y respeta en lo esencial la interdependencia, la heteronomía que lo define. Esta imposibilidad de encontrar sentido más allá de esas relaciones, de salir de la zona de seguridad del juego social y teórico dado, da buena cuenta del problema que queremos plantear aquí. Para hacerlo acudimos al más prevenido pensador contra las dicotomías, Pierre Bourdieu, en cuyos trabajos encontramos conceptos alternativos que permiten pensar el mundo social allende dichos pares.

\section{EL CONCEPTO CENTRAL DE HABITUS}

Loïc Wacquant ha definido su sociología como "una ontología social no cartesiana que rechaza la división entre sujeto y objeto, intención y causa, materialidad y representación simbólica" (Bourdieu y Wacquant, 2008: 134). La solución de la que deriva este rechazo del cartesianismo es particularmente interesante en lo tocante a los cuerpos y las almas. Bourdieu va a considerar que "los cuerpos y los cerebros son producto de la interacción social, más exactamente, de la apropiación de las y los 
agentes por parte del grupo social" (Bourdieu, 2008: 117). Y esa apropiación es la resultante de un proceso donde la doxa de ese grupo, en particular, es somatizada. La doxa no es aquí un conjunto de creencias transmitidas como un saber que expusiera, ya sea de manera explícita o implícita, las reglas culturales de esa sociedad; de modo que entre esta y sus miembros intermediara lo que habitualmente entendemos por aprendizaje. Como veremos, no hay consciencia del mismo, no hay adquisición de conocimientos, ni imitación de un modelo.

La doxa es descrita en la teoría bourdiana como "la realidad del orden del mundo, con sus sentidos únicos y sus direcciones prohibidas, en el sentido literal o metafórico"(Bourdieu, 2000: 11), que se reproduce en el cuerpo a cuerpo de la socialización. Lo que las y los agentes del grupo incorporan es una forma de existencia social, un cuerpo de creencias illo tempore sedimentadas en la acción en la forma de un sistema de disposiciones corporales (hexeis): un cuerpo que juega, come, anda, baila y se mantiene de cierta manera.

"El cuerpo cree en aquello a lo que juega: llora si imita la tristeza. No representa aquello a lo que juega, no memoriza el pasado, actúa el pasado, anulado así en cuanto tal, lo revive. Lo que se ha aprendido con el cuerpo no es algo que una/o tiene, como un saber que se puede sostener ante sí, sino algo que una/o es" (Bourdieu, 2008: 118).

El desarrollo corporal y cerebral de las niñas y los niños del grupo ocurre en este primer juego que es la mímesis (mimetismo) corporal de las disposiciones "que, en cuanto "hacer como si", implica una relación global de identificación" (Bourdieu, 2008: 118). Jugar aquí tiene un sentido práctico, operacional. "Hacer como si” es hacerse con ese mundo, identificarse como un/a jugador/a de ese juego al que ya siempre se está jugando.

Con la intención de proporcionar una comprensión intuitiva a sus lectoras/es de la noción de campo, Bourdieu la compara con un juego, pero cuya creación no es deliberada, ni sus reglas son explícitas. "Las y los jugadores son admitidas/os en el juego, se oponen unas/os a otras/os, algunas veces con ferocidad, solo en la medida en que coinciden en su creencia (doxa) en el juego y en lo que se juega, a lo que atribuyen un reconocimiento fuera de todo cuestionamiento" (Bourdieu y Wacquant, 2008: 135). La mímesis práctica ocasiona esa relación dóxica con el juego, que implica que no haya un afuera del juego desde cual cuestionarlo, sino únicamente un adentro donde jugador/a se reconocen como tales.

Abandonando el dualismo que relaciona al sujeto con el objeto, Bourdieu caracteriza esta relación como de "complicidad ontológica y de posesión mutua" (Bourdieu y Wacquant, 2008: 46), entre el sistema de disposiciones, habitus, y el mundo social que lo genera. De ahí, que tenga lugar un reconocimiento fuera de todo cuestionamiento, jugador/a y juego no se complementan sino que se identifican. El mundo social comparece así como un mundo cargado de sentido, un mundo compartido de sentido común. 
El campo es el espacio de posiciones definidas por relaciones objetivas de poder. "Las disposiciones adquiridas en la posición ocupada implican una adaptación a esta posición, lo que Goffman llamaba sense of one's place" (Bourdieu, 1988: 131). Identificarse con el mundo social equivale a interiorizar esa posición que se ocupa en el espacio relacional y que "se presenta, objetivamente, como un sistema simbólico que está organizado según la lógica de la diferencia, de la distancia diferencial" (Bourdieu, 1988: 137). Una posición en el campo solo puede definirse en relación a las otras, en relación a la red de relaciones objetivas entre esas posiciones. "El habitus implica un sense of one's place, pero también un sense of other's place"(Bourdieu, 1988: 134).

Pero, para comprender la noción de campo, es necesario introducir asimismo la de capital. "Una especie de capital es aquello que es eficaz en un campo determinado, tanto a modo de arma como de asunto en juego en la contienda, que permite a sus poseedores disponer de un poder, una influencia y, por tanto, existir en el campo en consideración, en lugar de ser considerada/o una cifra desdeñable" (Bourdieu y Wacquant, 2008: 136). Luego, las posiciones en ese espacio relacional están objetivamente determinadas por lo que Bourdieu denomina "los poderes sociales fundamentales que son, según mis investigaciones empíricas, el capital económico, bajo sus diferentes formas, el capital cultural, y también el capital simbólico, forma que revisten las diferentes especies de capital cuando son percibidas y reconocidas cómo legítimas" (Bourdieu, 1988: 131).

Un campo es siempre un espacio de posiciones de poder, un campo de poder. La posesión de capital simbólico asociada a una posición proporciona poder simbólico, el poder de legitimar el orden social. En todo campo tiene lugar una lucha simbólica por la producción de esa nominación legítima, por la producción del sentido común, de la verdad del mundo social. "El poder simbólico, en ese sentido es un poder de worldmaking" (Bourdieu, 1988: 140).

El juego mimético es el primer mundo, primer campo, las primeras experiencias configuran la primera realidad social. Esa realidad está ya siempre simbólicamente cargada, estructurada por el pasado. En cierto modo, nada es nuevo, sino renovado. La mímesis es un proceso de "reactivación práctica, se opone tanto a un recuerdo como a un saber, tendiendo a cumplirse más allá de la conciencia y de la expresión" (Bourdieu, 2008: 118). "Una/o no imita "modelos" sino las acciones de los otras/os" (Bourdieu, 2008: 118). La hexis corporal "es una manera práctica de experimentar y de expresar la opinión, como suele decirse, que se tiene de su propio valor social" (Bourdieu, 1998: 484). La incorporación de los esquemas de percepción y de apreciación asociados a una posición, a un habitus, expresan el estado del campo, es decir, "el estado de las relaciones de poder simbólico" (Bourdieu, 1988: 136). Como en un laboratorio, las disposiciones proporcionan el instrumento de medida de lo real, haciendo posible el experimento práctico. Pero, al mismo tiempo, limitan esa experiencia, ajustando sus variables a los valores recibidos, al estado de las relaciones de poder simbólico. El sentido práctico como sentido del juego es "sentido de los límites, anticipación práctica de los límites objetivos adquirida mediante la anticipación de los límites objetivos...de aquello de lo que se está excluida/o" (Bourdieu, 1998: 482). 
El primer juego social está lejos de ser la experiencia espontánea e inocente que aparenta. Si la doxa es necesaria, es porque sin ella el mundo es inexperimentable, inexpresable, no tiene límites y, sin límites, no hay orientación posible. La experimentación práctica está siempre limitada, dibuja, realizándose cada vez, eso que Virginia Woolf denomina las líneas de demarcación mágica, el mapa incorporado con sus senderos y sus precipicios, sus posibles y sus imposibles que permiten o prohíben desde el origen social este o el otro itinerario, el ascenso o el descenso, la inclinación hacia estas o las otras posibilidades.

"El habitus que en función de las estructuras producidas por las experiencia anteriores, estructura en cada momento las experiencias nuevas (...) realiza una integración única, dominada por las primeras experiencias..." (Bourdieu y Wacquant, 2008: 98).

Actuando el pasado, las niñas y los niños garantizan la perpetuación del grupo social, de las posiciones distintivas en el campo de poder, volviéndolo presente y futuro. Si las disposiciones pueden orientar socialmente la práctica es porque poseen la unidad de un estilo, de una maniera, porque están organizadas, reguladas por "la milenaria aplicación de los mismos esquemas de percepción y de acción que, no habiéndose constituido jamás en principios explícitos, no pueden producir sino una necesidad no buscada" (Bourdieu: 2008, 28). Ese estilo integra las experiencias de las y los agentes bajo la forma de limitaciones, prohibiciones, obligaciones que son como leyes prácticas incorporadas en el cuerpo social colectivo e individual.

"El habitus no es otra cosa que esa ley inmanente, lex insita inscrita en el cuerpo por las historias idénticas, que es la condición no solo de la concertación de las prácticas sino de las prácticas de concertación" (Bourdieu, 2008: 96).

Esa ley inscrita en los cuerpos, del organismo hecho ley inmanente, sentido práctico, sistema de disposiciones, apropiación social de los cuerpos y los cerebros:

"No tiene nada ni de más ni de menos misterioso, si se piensa en ello, que aquel que confiere su unidad de estilo a todas las opciones que puede una misma persona, es decir, un mismo gusto, operar en los dominios más diversos de la práctica...No es posible dominar esta lógica excepto para aquel que está completamente dominado por ella, aquel que la posee pero al punto de estar totalmente poseído por ella, es decir desposeído" (Bourdieu, 2008: 28).

\section{LA VIOLENCIA SIMBÓLICA DE LA EDUCACIÓN SENTIMENTAL}

La lucha simbólica por el monopolio de la legitimación del orden del mundo implica el continuo ejercicio de la violencia, pero esa violencia no es únicamente la 
violencia física, que desde luego es puesta en juego cuando es necesario, es decir, cuando la violencia simbólica, inherente al campo, violencia que atrapa a las y los agentes en sus propios cuerpos, no es suficiente.

La definición del campo como espacio de relaciones de poder simbólico implica que atendamos, como ya ocurriera en el caso de la noción de capital, a una especie de violencia que funciona en el plano simbólico de esa lucha por el reconocimiento de lo que es legítimo, de lo que tiene sentido. Como tal, se trata de una violencia "insensible e invisible, violencia simbólica, que tiene lugar a través de los caminos puramente simbólicos de la comunicación o el conocimiento, más exactamente, del desconocimiento, del reconocimiento o, en último término del sentimiento" (Bourdieu, 2003: 12).

Decíamos que las y los agentes poseen el sentido práctico, en la medida en que se ven desposeídos de su lógica. Esos sentimientos y desconocimientos, recursos simbólicos que violentan la acción de los agentes, garantizan el desenvolvimiento social condicionado por el habitus. Pero no de un modo determinista, esas y esos agentes no son tomados aquí como meros soportes de la realización estructural. La función de la noción de habitus, así como la de illusio o estrategia, a las que nos acercaremos a continuación, es la de poner de nuevo la praxis en manos de las y los agentes. Como si estuvieran "troqueladas", las líneas de acción tienden a doblarse de acuerdo a las exigencias del habitus, según las líneas de demarcación mágica marcadas por el campo. Las y los agentes tienen más probabilidades de tomar esta o la otra decisión y de poner en práctica este o el otro comportamiento, de sentirse de este o el otro modo ante los acontecimientos. Pero, claro está, siempre podrían hacer otra cosa.

"La verdad es que nunca ha dejado de asombrarme ante lo que podría llamarse la paradoja de la doxa: el hecho de que la realidad del orden del mundo, con sus sentidos únicos y sus direcciones prohibidas, en el sentido literal o metafórico sea grosso modo respetado, que no existan más transgresiones o subversiones..." (Bourdieu, 2003: 11).

Sabemos ya de dónde procede ese respeto, la sumisión a los sentidos únicos y las direcciones prohibidas del orden establecido. Lo paradójico está en que procede de las y los agentes mismos. La necesidad con la que se reproduce el orden social, la producen las y los agentes cada vez que se someten a sí mismos a sus emociones, a sus sentimientos, a la violencia de la ley inscrita en sus cuerpos, que no es otra que la ley del mundo social. Lo que las y los agentes han incorporado, como vimos, no son unos mandamientos explícitos, unas reglas, sino esa "necesidad no buscada, y por ende necesariamente imperfecta, pero también un poco milagrosa, y muy próxima en este sentido a la de la obra de arte" (Bourdieu, 2008: 28).

Ese carácter milagroso se opone tanto a la consciencia de estar siguiendo unas reglas que se tienen ante sí, como a un impulso inconsciente, al que se pudiera acceder por hipnosis. "No podemos comprender la violencia y la práctica simbólica sin abandonar por completo la división académica entre coerción y consentimiento, 
imposición externa e impulso interno"(Bourdieu y Wacquant, 2008: 218). Por un lado, las y los agentes no tienen conciencia de ser coaccionados a seguir unas reglas impuestas desde fuera; por el otro, tampoco responden a un impulso interno, espontáneo y natural. Recordemos que el habitus es el sentido del juego incorporado en cada una y uno de los agentes, vuelto naturaleza (Bourdieu, 1988: 70). Esa "naturalidad" un poco milagrosa y paradójica, forzosa, que domina a los agentes impidiéndoles llevar a cabo más transgresiones y subversiones, contiene, sin embargo, algo de ventaja, o mejor, de voluntad.

Cada campo, cada juego produce un tipo de interés específico. Para definirlo de manera muy sencilla, ese concepto de interés, que en la evolución de su teoría pasa a denominarse illusio y luego libido, es aquello que hace que el jugador, la jugadora estén concernidas/os, tomadas/os por el juego. "Estar interesada/o es aceptar que lo que ocurre en un juego social dado importa, que la cuestión que se disputa en él es importante...y que vale la pena luchar por ella" (Bourdieu y Wacquant, 2008: 156). Esa adhesión al juego, esa disposición libidinal que genera el habitus conduce "al despliegue activo de «líneas de acción» objetivamente orientadas que obedecen a regularidades y conforman patrones coherentes y socialmente inteligibles" (Bourdieu y Wacquant, 2008: 46). Bourdieu denomina a esas "líneas de acción", estrategias. No obstante, nos advierte de que no hay en su consecución ni intención premeditada ni estratega, sino la illusio, la libido, el interés que violenta la práctica de las y los agentes.

Loïc Wacquant explica que ese interés, esa illusio, libido, "quiere expresar la idea de que la gente es motivada, forzada, arrancada de un estado de in-diferencia y movida por los estímulos de ciertos campos y no de otros...porque no ha sido socializada para darles valor" (Bourdieu y Wacquant, 2008: 52). Como sabemos esa socialización ocurre en la primera infancia, durante las primeras experiencias. Los habitus "las/os sensibilizan y movilizan para percibirlos y perseguirlos" (Bourdieu y Wacquant, 2008: 53). La única posibilidad real para las y los agentes de participar en el juego social, es decir, de interesarse, de ilusionarse, de ser alguien y no nada, siquiera el elemento dominado, pasivo y perdedor es esa adhesión a un juego.

"La violencia simbólica se instituye a través de la adhesión que la/el dominada/o se siente obligada/o a conceder al dominador/a (por consiguiente a la dominación), cuando no dispone, para imaginarla o para imaginarse a sí misma/o o, mejor dicho, para imaginar la relación que tiene con él/ella, de otro instrumento de conocimiento que aquel que comparte con el dominador/a y que, al no ser más que la forma asimilada de la relación de dominación, hacen que esa relación parezca natural” (Bourdieu, 2003: 51).

Adherirse al juego implica adherirse a sus relaciones y con ella a su polo dominante y ello aún a costa de desadherirse, de minusvalorarse a sí mismas/os, es decir de soportar, de alimentar la violencia simbólica que implica esa relación. Desacreditar el juego, desacreditar al dominante supone minar esa relación y equivale a desacreditarse una/o misma/o. De ahí, que la cadena de transmisión de esa violencia invisible, tienda a seguir la dirección única de la dominación simbólica. Para evitar 
subvertir el sentido del juego, el eslabón dominado soporta o bien transmite hacia posiciones análogas, o aún más dominadas, la violencia simbólica que se ejerce sobre él. De esta forma, no perturba su posición ni la relación que le distingue, manteniendo la distancia sentimental, pasional y emocional que les diferencia de las y los otras/os, no solo de las y los dominantes sino de las y los dominadas/os. La reproducción de un estado determinado del campo, el mantenimiento de los límites y de las relaciones de distinción implica por parte de las y los agentes, violentarse a sí mismas/os y necesita de un trabajo continuo de separación física y moral, disposicional y de segregación social. Todo ello inversión por excelencia de la dominación masculina.

\section{LA DOMINACIÓN MASCULINA}

Sobre esa realidad biológica que son los cuerpos, se construye toda una realidad social que radica en "una topología sexual del cuerpo socializado, de sus movimientos y de sus desplazamientos inmediatamente afectados por una significación social" (Bourdieu, 2003: 20). Se construyen unas diferencias jerárquicamente organizadas, que somatizadas como disposiciones corporales, son percibidas cómo naturales, naturalizando así la construcción social misma y la relación de dominación que la organiza.

Esa topología sexual del cuerpo socializado, radica, como ya sabemos en un cuerpo social topológicamente sexualizado. Deshistorizando y, por eso, naturalizando las condiciones sociales que posibilitan su comprensión, las inversiones en cierta idea de "cuerpo" y de "sexo", virtualmente abstraídas de sus condiciones sociales de producción, separadas de su genealogía, posibilitan que queden cargadas de una significación esencial, velada y misteriosa (que no es sino el resultado de la inversión entre las causas y los efectos), sobre la que se proyectan paradójicamente los esquemas de percepción de la sociodicea masculina que los originan.

"La paradoja consiste en que son las diferencias visibles entre el cuerpo femenino y masculino las que, al ser percibidas y construidas de acuerdo con los esquemas prácticos de la visión androcéntrica, se convierten en el garante más indiscutible de significaciones y de valores que concuerdan con los principios de esta visión del mundo" (Bourdieu, 2003: 36).

Hablar del cuerpo en sí mismo, es tan eficaz para la estructura de dominación como hablar de la sexualidad en sí misma. Afirmar que hay un cuerpo y un sexo como tales, es rehuir la naturaleza relacional y social de ambos conceptos y permanecer en el esencialismo que garantiza la pervivencia y la reproducción de los esquemas de la visión androcéntrica, que permanecen así impensados. Pero este desconocimiento, que posibilita la adhesión al juego afecta por igual a mujeres y varones. Si las jugadoras se sienten inclinadas hacia estrategias de adhesión, que garantizan su dominación estructural por parte de sus "compañeros" varones, es porque no saben lo que hacen, en el sentido "simbólico" de la expresión. Pero esto mismo puede aplicarse a los segundos. Son ellos quienes realizan esta... 


\begin{abstract}
"Inversión primordial en los juegos sociales (illusio)...principio indiscutido de todos los deberes hacia uno mismo...Esta inversión se halla en la relación entre un hábito construido de acuerdo a la división fundamental entre lo recto y lo curvo, de lo erguido y de lo abatido, de lo fuerte y de lo débil, en suma, de lo masculino y lo femenino, y un espacio social organizado también de acuerdo con esta división que engendra, como tantos dictadores, las inversiones agonísticas de los hombres y de las virtudes, todas ellas de abstención y abstinencia, de las mujeres" (Bourdieu, 2003: 67).
\end{abstract}

Habitus y espacio social están atravesados por esa división fundamental que posibilita la circulación de capital simbólico o, si se quiere, la transformación de cualquier especie de capital en capital simbólico, sentido del honor, virilidad. Las y los agentes son sensibilizadas/os y movilizadas/os por esa illusio androcéntrica, libido dominandi que es siempre el asunto en juego.

Las estrategias que las mujeres realizan para jugar propiamente a ese juego, para convertirse de "jugadoras en vías de desarrollo", en jugadoras de pleno derecho, desconocen la paradoja de perseguir esa illusio. Al estar orientadas por esa misma libido viril y por ello encaminadas a reforzarla, cualquier acción que no cuestione el juego, está destinada a alimentarlo. Pero ese cuestionamiento ha de tener en cuenta "la «contradicción irresoluble» inscrita en la lógica misma de la dominación simbólica. La resistencia puede ser alienante y la sumisión puede ser liberadora" (Bourdieu y Wacquant, 2008: 50). ¿Cómo salir de ella?

\title{
4. EL EXPERIMENTO WOOLFIANO COMO EVOLUCIÓN DEL JUEGO
}

Vamos a detenernos en esa "necesidad no buscada, y por ende necesariamente imperfecta, pero también un poco milagrosa, y muy próxima en este sentido a la de la obra de arte propia de la milenaria aplicación de los mismos esquemas de percepción y acción" (Bourdieu, 2008: 28). Quizá ese vínculo con la obra de arte pueda guiarnos en la tarea sumamente difícil, pero no imposible, de salvar la contradicción irresoluble de la lógica de la dominación masculina.

Dentro del juego, es complicado en extremo calibrar las posibilidades liberadoras de ciertas acciones. Las transgresiones inevitablemente implican el acato de los límites que se traspasan, precisamente porque es necesario suponerlos para traspasarlos. Quebrantar la ley, supone creer en ella, en el sentido que hemos venido explicando en la primera parte de este artículo. Por eso, la liberación dentro de la disciplina del campo, es liberación de una/o mismo, separación, alienación, transgresión y subversión respecto de los límites ínsitos en el propio cuerpo. 
"Al marcar solemnemente el paso de una línea que instaura una división fundamental del orden social, el rito atrae la atención del observador/a hacia el hecho del paso (de ahí la expresión rito de paso), cuando lo importante en realidad es la línea. ¿Qué separa, en efecto, esta línea?" (Bourdieu, 2001: 79).

Como en los ritos así llamados "de paso", lo importante para salvar esa contradicción en realidad no es pasar la línea, transgredir la ley, sino la línea de demarcación, la ley misma y la contradicción a la que aboca. Si la contradicción es insuperable es porque es consustancial al sentido del juego, porque la lex insita funciona como contradicción práctica. ¿Qué separa, en efecto, ese sentido del juego, esa lex insita en los cuerpos y los cerebros de las mujeres y los varones? Como en los ritos de paso, la ley separa los que son aptas/os para el juego de los que no lo son, quienes pueden porque deben tomarse el juego en serio de quienes no. Bourdieu los denomina ritos de institución, puesto que su función es "instituir así una diferencia constante entre aquellas/os a quienes concierne el rito y aquellos/as a quienes no concierne" (Bourdieu, 2001: 78). Es decir, lo que el ritual establece es "el límite entre lo lícito y la transgresión" (Bourdieu, 2001: 78). Dar ese paso, traspasar la línea que segrega los cuerpos y los cerebros masculinos de lo que no lo son ni pueden ser viriles, cuando a una no le está permitido, equivale indefectiblemente a una transgresión. Como viera con claridad Woolf hace ya casi un siglo, la participación de las mujeres en el juego masculino está cargada de ese carácter transgresivo, que hace que toda supuesta liberación dentro del juego, suponga un traspaso del ámbito de lo femenino al de lo masculino y, por lo tanto, tenga algo de ilícito no solo para el mundo social, sino para una misma. Sin duda, la alienación a la que conduce la contradicción es insuperable dentro del rito. Pero, ¿qué hay en el mundo social sino un conjunto de rituales de paso cuyo "mayor efecto es el de pasar completamente desapercibidos" (Bourdieu, 2001: 79).

"El lema de Josephine Butler -Justicia, Igualdad, Libertad- es hermoso pero es solo un lema y en nuestro tiempo hay innumerables lemas, lemas de todos los colores, y hemos llegado a contemplar con suspicacia los lemas, matan y limitan. La vieja palabra libertad no nos sirve tampoco, debido a que no era libertad en el sentido de permiso lo que querían, al igual que Antígona no quería quebrantar las leyes sino hallar la ley" (Woolf, 1999: 241).

La dominación masculina aboca a las mujeres a una práctica de liberación de dirección única, de abajo hacia arriba, de lo femenino a lo masculino. De ahí el sentido de permiso del que habla Woolf, que comparte con Foucault, la desconfianza "ante el tema general de la liberación...esta práctica de liberación no basta para definir las prácticas de libertad que a continuación serán necesarias" (Foucault, 1999: 394). En efecto, subsiste aún la relación diferencial que les define. "Hay un conjunto escondido con relación al cual se constituye el grupo instituido" (Bourdieu, 2001: 79). Lo que el acceso de las mujeres a las posiciones masculinas del espacio social avala es un 
reforzamiento de la creencia, de la libido dominandi, del agón como asunto en juego. Las prendas exigidas a las mujeres para ocupar esos lugares intensifican el esencialismo universalista, que hace pasar las aptitudes y actitudes masculinas como necesarias y universales, el conjunto escondido con relación al cual se constituye el grupo instituido.

"Para cambiar el mundo, es necesario cambiar las maneras de hacer mundo, es decir la visión del mundo, y las operaciones prácticas por las cuales los grupos son producidos y reproducidos" (Bourdieu, 1988: 140).

Lo que Woolf propone es un activismo tan indiferente como creativo, hay que cambiar la manera de hacer mundo, haciendo mundo y ello no será posible mientras sigamos creyendo y otorgando valor a cómo es. Hemos de fijar nuestra visión no en el paso, sino en la línea, en los límites objetivos que la estructura produce y en la contradicción irresoluble al que las mujeres se ven arrojadas cuando no lo hacen. Hacer mundo es redibujar esa línea en una misma, estar dispuestas a algo mucho más difícil y arriesgado que quebrantar la ley, hallar la ley, hacer mundo primero con el cuerpo propio. Hacernos un cuerpo propio.

"Virginia Woolf nos permite entender cómo al abandonar la illusio que lleva a uno/a a participar en los juegos centrales de la sociedad, las mujeres escapan a la libido dominandi que dicha participación trae consigo y, por tanto, tienen una predisposición social que les permiten una visión relativamente lúcida de los juegos masculinos, de los que habitualmente participan solo por proximidad" (Bourdieu y Wacquant, 2008: 219).

El punto débil de la dominación está en esa predisposición social de las mujeres hacia la lucidez cuyo fin es, para Woolf, una actitud de total indiferencia (Woolf: 1999, 187). Abandonar esa illusio por proximidad y volverse hacia el juego con una visión completamente ajena, forastera, outsider conlleva revolucionar las relaciones entre los habitus y el campo. En efecto, si "la noción de interés se opone a la de indiferencia" (Bourdieu y Wacquant, 2008: 155), es porque la distancia diferencial, la ley del campo y de los habitus queda anulada, cada vez que la creencia se suspende.

"Hablando en términos generales la principal distinción entre nosotras, entre las que por estar fuera de la sociedad, somos outsiders y los que, por estar dentro de ella son naturales, entre las que estamos fuera y los que están dentro, será que mientras ustedes harán uso de los medios suministrados por su posición -coaliciones, simposios, campañas, grandes nombres y todas aquellas medidas públicas que su riqueza y política influencia ponen al alcance de sus manos -, nosotras que seguimos siendo outsiders, haremos experimentos. Pero no con los medios públicos, en público, sino con los medios privados en privado. Estos experimentos no serán meramente críticos sino creadores" (Woolf, 1999: 199). 
Esa suspensión de la creencia solo puede producirse gracias a la vigencia de una nueva ley, la que esos experimentos no solo críticos sino creadores generan, cada vez que se realizan. Realizar experimentos es hacer mundo, hacer una visión, una interpretación del mundo, de los cuerpos y de los cerebros mediante la cual las y los agentes se reapropien de sí, de sus habitus.

Volviendo a Foucault, llevar a cabo prácticas de libertad no implica quebrantar, transgredir la ley sino encontrar la propia, "que esos individuos puedan definir formas válidas y aceptables, tanto de su existencia como de la sociedad política" (Foucault, 1999: 395). No hay permiso ni transgresión, sino decisión y acción, al margen de los valores dados.

Sabemos ya que todo habitus es por definición insider, generado en el seno de un campo social dado y asociado a una posición que queda definida en relación al resto de posiciones de ese espacio diferencial. Asumir la condición de outsider no implica dejar de pertenecer a ese campo, dejar de tener una posición, un habitus, sino convertirse en relación a él en un punto ciego, no sensible a la "luz simbólica" que emana del mismo. No obstante, la manera de cegarse, de insensibilizarse consiste en una actividad creadora constante. Lejos de ser pasivo, el habitus outsider no puede dejar de experimentar, de investigar la ley que cada vez crea nuevos valores, generando las condiciones de posibilidad de un habitus propio. De ahí que sea "tan importante acostumbrarnos a los deberes de la libre expresión, puesto que sin libertad privada no puede haber libertad pública" (Woolf, 1999: 210).

Luego lo que Woolf plantea es una libre expresión en carne propia, que aprovecha la plasticidad de los cuerpos y los cerebros, que quedaba probada en el proceso de mimetismo mediante el que el grupo se apropiaba de los mismos. Esa prueba nos respalda para lo que proponemos, volver sobre sí, sobre el habitus adquirido, conocerse a sí misma/o, al campo no solo crítica sino creativamente. No solo poniendo en cuestión, sino inundado con experimentos aquellos caminos simbólicos del desconocimiento y del sentimiento. No hay plan ni, por lo tanto, estratega para el mismo. A pesar de esta ausencia de causa final, los efectos se multiplican, proliferan rompiendo las dicotomíasque nutren la dominación.

\section{HABITUS DADÁ}

En la obra de arte: "la traducción sensible oculta la estructura, en la forma misma en la que se presenta y gracias a la cual logra producir un efecto de creencia(más que de realidad)...lo dice de tal modo que no lo dice de verdad" (Bourdieu, 1995: 63).

Cuando más arriba hablamos del mimetismo primario, explicamos cómo la estructura quedaba velada por el proceso mismo de mimesis corporal, al que asimismo podríamos referirnos cómo una traducción sensible que oculta la estructura, en la forma misma en la que se presenta y gracias a la cual logra producir la creencia. Por otro lado, también la obra de arte es en cierto modo un "hacer como si", hacer como si fuera verdad, hacer creíble, verosímil lo que no es realidad, sino ficción. Ese efecto de 
creencia se basa en "la coincidencia entre las suposiciones que la obra introduce y las que introducimos en la experiencia habitual del mundo" (Bourdieu, 1995: 63).

Recordemos que esas suposiciones que introducimos en la experiencia habitual del mundo, fueron mimetizadas en la primera infancia y que es en calidad de tales que se corresponden con la "realidad". Además, esta correspondencia se basa igualmente en una coincidencia, esta vez entre las suposiciones incorporadas, aquellas disposiciones que vienen actuándose desde un pasado remoto, y las suposiciones del mundo social. Por todo ello, podemos afirmar que unas y otras suposiciones, las del habitus y las de la obra, son productos contingentes, creaciones, obras que operan del mismo modo, buscando la coincidencia entre suposiciones, aquella que generaba esa necesidad no buscada y casi milagrosa que Bourdieu encontraba común a la obra de arte y al sentido práctico.

En Las Reglas del Arte, Bourdieu analiza una obra literaria específica La educación sentimental de Gustave Flaubert, considerando que "restituye de forma extraordinariamente exacta la estructura del mundo social en el que ha sido elaborada...Pero lo hace con los medios que le son propios, es decir, haciendo ver $y$ sentir con ejemplificaciones o, mejor aún, evocaciones, en el sentido fuerte de hechizos capaces de producir unos efectos, particularmente sobre los cuerpos" (Bourdieu, 1995: $63)$.

Los cuerpos y los cerebros que han incorporado ese sentido práctico, los habitus, creen en aquello a lo que juegan, lloran cuando imitan la tristeza. Los habitus son esos cuerpos hechizados sobre los que el proceso de mimesis ha causado profundos efectos, dibujando sobre ellos aquellas líneas de demarcación mágica de las que hablara Woolf. Y lo ha hecho haciéndoles ver y sentir, evocando el pasado, reviviéndolo, produciendo en ellos esos efectos de creencia, como si se tratara de una antiquísima forma de arte sin autor o autora, colectiva y anónima.

Los cuerpos y los cerebros podrían, en efecto, ser considerados como el material más precioso con el que una o un artista pudiera trabajar. Como Tres Guineas de Virginia Woolf, las “obras” del llamado movimiento Dadá ocurren cuando una Guerra Mundial hace que la potencia de movilización de la libido dominandi alcance cotas nunca antes alcanzadas. Cabe decir que sus integrantes guardaban también una actitud de total indiferencia hacia la Guerra, no comprendiendo en absoluto qué viril satisfacción reportaba la lucha. Eso explica seguramente su huida a Zurich, donde Hugo Ball dió acta fundacional al movimiento al fundar en 1915 el Cabaret Voltaire.

Dadá va a intervenir en el mundo social considerando los habitus de las y los agentes el material por definición a transformar. Sus acciones son experimentales, colectivas e individuales, ni privadas ni públicas sino todo lo contrario. "Se recurrió al mal gusto, a la sorpresa, el escándalo...El gesto sustituirá la mayoría de las veces al objeto y el arte, en sus manos, se convertirá en un juego para acabar con el Arte con A mayúscula" (Aracil, Rodríguez, 1998: 192). El juego Dadá no acudía, como ocurrirá con las integrantes de la society of outsiders, a los medios habituales. Tampoco se restringía ni mucho menos al juego artístico, porque el juego Dadá abarcaba la totalidad del campo social. Acabar con el Arte, era también acabar con la Moral y con 
la Política, entre otras. En el Primer Manifiesto Dadá, TristanTzara manifiesta: "Estoy por principio contra los manifiestos, como también estoy contra los principios" (Aracil, Rodríguez, 1998: 193).

Los experimentos creadores de Woolf y las acciones del movimiento artístico Dadá, tienen en común un deseo de ruptura que no busca ser meramente crítico sino creativamente anclado a eso que Woolf denomina la realidad, que Tzara denomina la vida y que Bourdieu denomina habitus. Primero, se trata de guardar un momento de total indiferencia o, si se prefiere, de nihilismo absoluto: no hay que comprender nada, nada, nada, solo los valores que tú misma/o darás a todo (Aracil, Rodríguez: 1998, 193). En esa nada se incluyen sin duda, "los juegos que se suponen cruciales para la sociedad, como los juegos de honor y guerra (adecuados para el despliegue de masculinidad, virilidad) o en las sociedades avanzadas, todos los juegos más valorados como la política, los negocios, la ciencia, etc." (Bourdieu y Wacquant: 2008, 218). Hay que convertirse en un punto ciego para el campo. Segundo, es imperativo iniciar la búsqueda de la ley o, si se quiere, de la creación de valores, consumar el experimento, la acción dadaísta, comprendiendo solo los valores que tú misma/o darás a todo. Y todo ello, secretamente, ni pública ni privadamente, porque "todavía debemos ocultar lo que hacemos y lo que pensamos, a pesar de que lo que hacemos y lo que pensamos promueve una causa común" (Woolf, 1999: 209). Teniendo como rango de acción (de anclaje, dice Woolf) la realidad, la vida, el habitus. Se trata de imaginarnos, de crearnos de otra manera, de hacerse un habitus dadá, un habitus outsider.

La escritura de este artículo quiere ser uno de estos experimentos.

\section{REFERENCIAS BIBLIOGRÁFICAS}

ARACIL, A., RODRÍGUEZ, D. (1998): El siglo XX. Entre la muerte del Arte y el Arte moderno. Madrid: Istmo.

BOURDIEU, PIERRE (1988): Cosas dichas. Buenos Aires: Gedisa.

BOURDIEU, PIERRE (1995): Las Reglas del Arte. Génesis y estructura del campo literario. Madrid: Anagrama.

BOURDIEU, PIERRE (1998): LaDistinción. Madrid: Taurus.

BOURDIEU, PIERRE (2003): La Dominación masculina. Barcelona: Anagrama.

BOURDIEU, PIERRE (2001): ¿Qué significa hablar? Economía de los intercambios lingüísticos. Madrid: Akal.

BOURDIEU, P.(2008): El sentido práctico. Madrid: Siglo XXI.

BOURDIEU, P., WACQUANT, L. (2008): Invitación a una sociología reflexiva. Buenos Aires: Siglo XXI.

FOUCAULT, M. (1999): Estética, Ética, Hermenéutica. Barcelona: Paidós.

WOOLF, V. (1999): Tres Guineas. Barcelona: Lumen. 